\title{
The use of computers in high energy physics experiments
}

D Lord and G R Macleod

Data Handling Division, CERN, Geneva, Switzerland
Abstract An introductory description is given of the nature of high energy physics experiments and of the characteristics of the detectors employed; the main uses of computers in this field are briefly outlined. A more detailed review is made of the rôle of computers in film measurement and data taking methods, indicating the limitations set by present computing techniques and some future trends to be expected.

\section{Outline of high energy physics experiments}

\subsection{Introduction}

The aim of high energy physics research is to measure the properties of the so-called elementary particles and to determine the nature of the forces to which they are subject. The principal tools used in this field are a number of particle accelerators-proton and electron synchrotrons or linear accelerators - capable of producing controlled beams of high energy particles with energies in the range of $1-70 \mathrm{Gev}$ (Howard 1967a). These accelerators are large, complex and expensive items of equipment and there are about a dozen national and international laboratories which operate them (Howard 1967b).

A typical high energy physics experiment involves bringing a beam of particles of known kind and energy on to a target (often liquid hydrogen which provides a comparatively high concentration of protons) and observing the secondary particles produced within the target volume. They may be produced either by collisions between the incident particles and the nuclei in the target material or by radioactive decays of unstable particles. The trajectories and momenta of the incident and secondary particles may be determined by the use of various detectors and magnetic fields. An experiment may involve the measurement and analysis of hundreds to hundreds-of-thousands of such nuclear events. The analysis of an event often involves long and complex calculations and to repeat these many times for a large number of events was the earliest reason why computers were used in this field. It is to-day still the largest single use of computers in high energy physics.

\subsection{Detectors used}

Three main types of detectors are used, scintillation and Cerenkov counters (Piroué 1966), bubble chambers and spark chambers (Ritson 1966). Scintillation counters simply detect the passage of a charged particle with time resolutions in the range 10-1ns. Their dead-times (i.e. the time for which they remain insensitive after the passage of a particle) are equally small. They generally operate by a photomultiplier detecting the small light pulse produced when a particle traverses a suitable material. Scintillation counters use NaI crystals or transparent plastic with a scintillating additive, whilst Cerenkov counters use a gas under pressure. These counters can

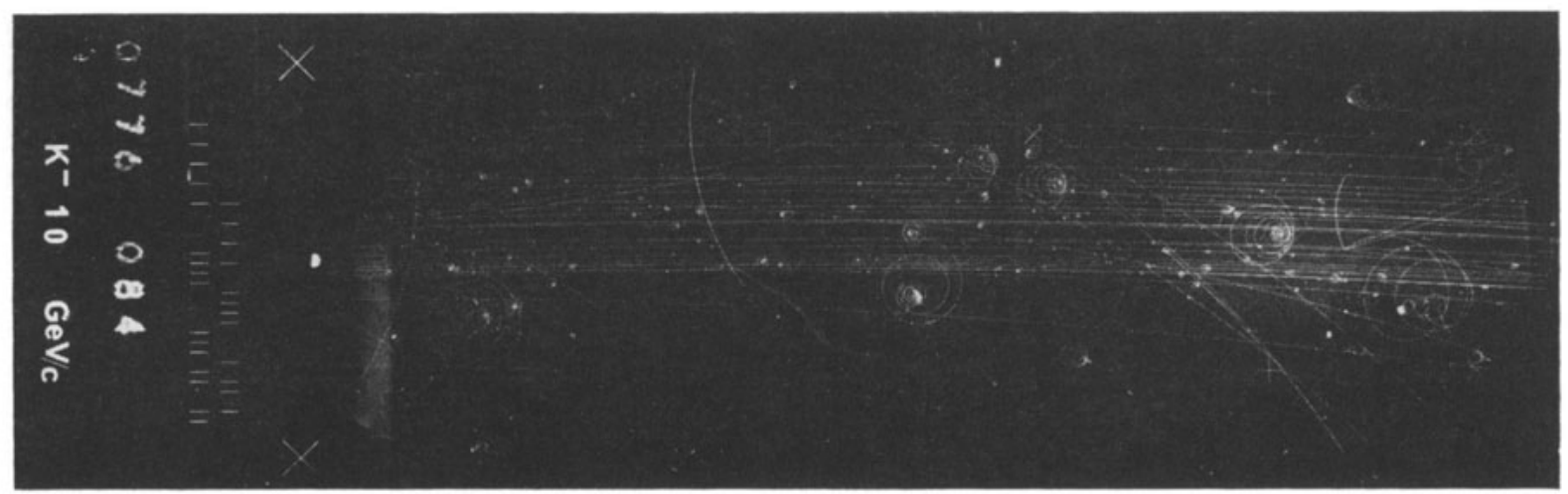

Figure 1 A hydrogen bubble chamber photograph with a beam of $10 \mathrm{Gev} / \mathrm{c} \mathrm{K}^{-}$mesons. The beam tracks which enter the chamber from the left, are the long slightly curved parallel tracks going the whole length of the picture. An interaction can be seen close to right hand side with five visible secondary tracks. The various crosses are fiducial marks and the alphabetic and binary coded identification data can be seen at the left-hand side in the 'data box' 


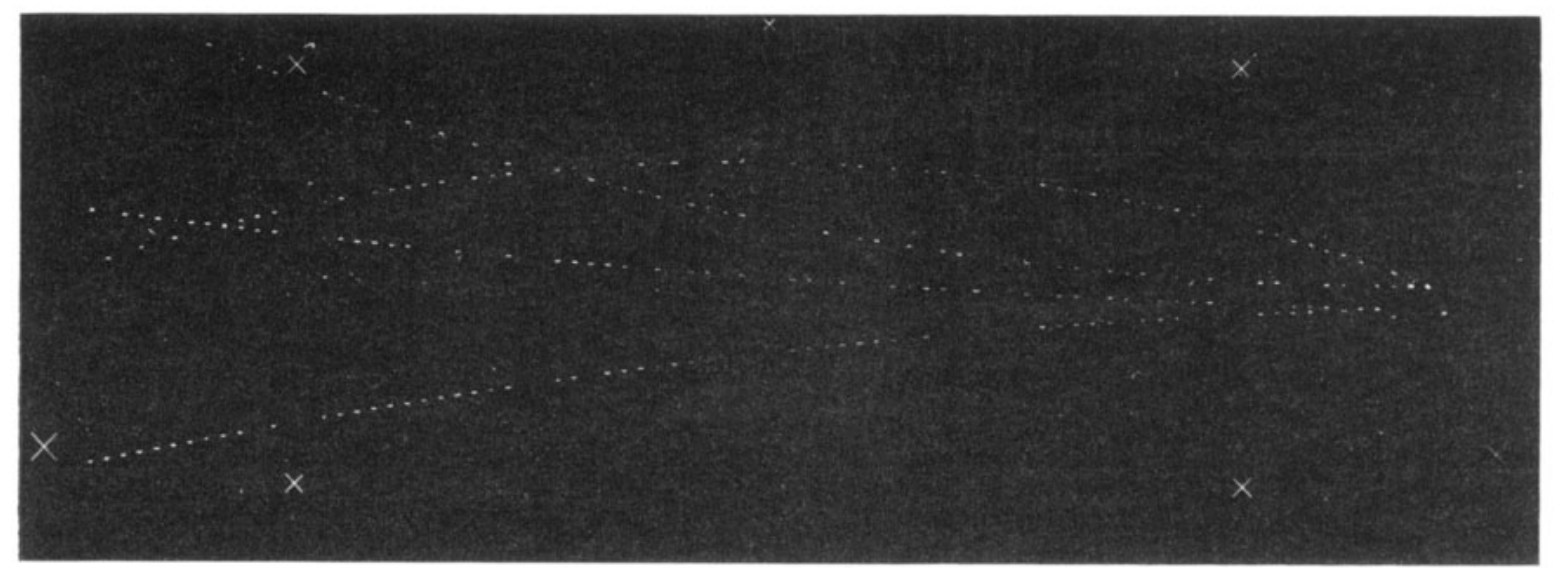

Figure 2 A photograph from a multi-gap spark chamber, operating in a magnetic field of $10.5 \mathrm{~kg}$, showing tracks of simultaneous decay of two $\mathrm{K}^{\circ}$ mesons

achieve counting rates of $10^{i}-10^{8}$ per second. In practice counting rates are more limited by the speed of the associated counting circuitry rather than the counters themselves. Cerenkov counters, whose counting characteristics are similar to those of scintillation counters, are used for detecting particles of a certain velocity. The physical dimension of the sensitive area of scintillation counters can vary from square millimetres to a square metre or so. Large arrays of counters arranged in a checkerboard pattern (hodoscope arrays) have been used (Foley et al. 1964) to measure the position of particle trajectories. However, generally speaking, counters are mainly used to detect the passage of particles; they can readily measure particle position to within a centimetre or so, but as the precision required increases much beyond this, the complexity of the counter arrangement rises enormously.

The geometrical arrangement of counters in the incident beam and around the target, together with the time coincidence criteria imposed upon their counts, can be used to select the kind of event being examined from all the other background counts caused by beam particles which do not interact in the target, stray radiation from the accelerator, etc. Such a selection mechanism can also be used to operate or to 'trigger' other detectors in the system only when events of the required kind are thought to be present, thus avoiding recording large amounts of useless background data. Information from counters is extracted by using a variety of equipment such as coincidence circuits, scalars and pulse height analysers. Final storage of the information may be made on notebooks, teleprinters, punched tape or cards, or magnetic tape depending on the quantity of data and the complexity of the measurements being made.

Bubble chambers are detectors which enable a photographic record to be made of particle trajectories and of events which occur by collision of the beam particles with nuclei of the chamber liquid, usually liquid hydrogen or propane. They operate by making a rapid expansion of a supercooled liquid as the beam particles traverse the chamber. This causes local boiling along the ionized tracks left by the particles. An electronic flash then illuminates the bubbles so formed (which are about $1 \mathrm{~mm}$ in diameter) and images of the particle tracks are recorded, usually on three or four stereoscopic photographs. Stereoscopic photographs are taken so that by suitable measurements and calculations it is possible to determine the position and direction of the tracks in space. Chambers up to $2 \mathrm{~m}$ long and about $0.5 \mathrm{~m} \times 0.5 \mathrm{~m}$ in section are in operation. They are sensitive for some $100 \mathrm{~ms}$ after the expansion and they can usually be expanded every few seconds when the pulsed particle beam produced by the accelerator traverses the chamber. Each expansion produces one set of three stereo photographs or 'triad'. The chamber is in a magnetic field (up to $20 \mathrm{~kg}$ ) so that the particle trajectories are curved, the curvatures being a measure of the particle momenta. The number of bubble images per unit length of a track is related to the ionization caused by the particle. In addition to the images of the tracks, bubble chamber pictures contain images of fiducial marks and of 'data-box' information. Fiducial marks are usually crosses engraved on the windows of the chamber whose position in space is accurately known, and they provide the means by which the coordinate system in which the film is measured can be related to the real spatial coordinates. The data-box displays identification information: serial number of the photographs, experimental conditions, date, etc. This information is usually in a legible form as it has to be read by human beings, but may also be repeated in a coded binary form to be read by automatic machines.

By their nature, bubble chambers cannot be triggered and so they record all tracks which pass through their volume. A typical experiment may take several tens- to hundreds-ofthousands of triads, all of which have to be visually examined (pre-scanning) $\dagger$ to select those containing events of interest. Those selected, maybe several hundreds to several thousands, have to be measured and analysed (figure 1).

Spark chambers can be used either as counters with a high spatial resolution or as devices which, like bubble chambers, 'realize' events by recording images of particle tracks on stereo photographs. They operate by applying a high voltage pulse (tens of kilovolts for a few microseconds) to two electrodes in an inert gaseous mixture, just after the passage of a particle. The ionized track left by the particle causes an electrical breakdown and the spark indicates the position of the track. The electrodes can be plane parallel sheets of foil (e.g. aluminium, $10-25 \mu \mathrm{m}$ in thickness) about $1 \mathrm{~cm}$ apart. There can be many such planes, and alternate ones are pulsed to many kilovolts whilst the others are held at earth potential. These are the so-called multi-gap spark chambers. This type of chamber is usually photographed, the long succession of

† We use this term rather than the more usual 'scanning' in order to distinguish it from the other sense of the term scanning used in section 2 which is the detailed examination of film with a spot of light or a light sensitive device. 
sparks indicating the particle trajectory (figure 2). Usually two stereo photographs are taken, again to allow the position of the sparks in space to be determined. The two electrodes can also be planes of parallel wires with about $1 \mathrm{~mm}$ separation between wires, and adjacent planes can be arranged to have their wires running in orthogonal directions to each other. When a particle causes a spark between the electrodes, the discharge current flows in one wire in each plane, thus locating the spark position to about $\frac{1}{2} \mathrm{~mm}$. These are called wire chambers. Spark chambers with electrodes of over a metre square are in operation with electrode separations of $\frac{1}{2}-1 \mathrm{~cm}$. They are triggered, i.e. the high voltage is applied, only if some other counter arrangement indicates that it is very likely that an interesting event has occurred. The shortness of the applied voltage pulse ensures that these chambers are sensitive for only a few microseconds, and so sparks are formed only by particles passing through the chamber at the same time as the triggering event. This greatly reduces the number of background tracks. With spark chambers, particle positions can be measured to a precision of about $\frac{1}{2} \mathrm{~mm}$ in $1 \mathrm{~m}$. The dead-times are in the range of $1-10 \mathrm{~ms}$; when photographic recording is used this increases to some $50 \mathrm{~ms}$ or more, being determined by the film advance speed in the camera. A typical spark chamber experiment may take several hundreds-of-thousands of photographs, most of which have to be measured, since the triggering system has prevented most of the unwanted events from being recorded at all.

\subsection{Areas of computer use}

Computers are used in the planning, data acquisition and data analysis phases of high energy physics experiments, as well as in control functions for accelerators (Howard 1967a, beam switchyards (Howry 1967) and bubble chambers (Simpson 1967). In planning an experiment simulation calculations can be made by using Monte-Carlo techniques (James 1968) for estimating event rates to be expected, counting rates due to background, optimum disposition of detectors and so on. Much beam optics design (Whiteside and Gardner 1963) is done by computing particle trajectories through complex systems of magnets and changing various parameters to obtain the best calculated performance. These results are then used in setting up the beam for an experiment.

In the data acquisition phase of an experiment, computers are used on-line to electronic detection equipment (counters, spark chambers and their associated electronics) to record the experimental data in digital form and to assist in checking the correct functioning of the apparatus ( $\S 3.1,3.2$ ). In bubble chamber experiments, computers are not used to collect or analyse data during the accelerator runs, although they may be used to log or to control the operating conditions of the chamber. For subsequent measurement of the film, however, various special purpose devices have been built, many of which are now operated on-line to computers ( $\$ 2.1,2.2$ ). This measurement procedure is essentially a complex analogue-to-digital conversion in which the computer plays a control and processing rôle, transforming the experimental (analogue) data on film into digital form.

In both kinds of experiments the computer is also used to reduce the volume of experimental data before passing to the data analysis phase, where detailed calculations are made on the events to be examined. In counter experiments the distinction is not always clear; the on-line computer program sometimes carries out the data reduction as the data is acquired, while sometimes the analysis programs reject a significant amount of the recorded data at a very early stage in the processing, based on simple topological, geometrical or kinematic criteria. In bubble and spark chamber experiments when the film is measured by automatic film measuring devices, the data reduction is frequently carried out by so-called 'pattern recognition' or 'filter' programs. Based on topological criteria alone, these programs select the measurements which correspond to the pattern of tracks of the event to be analysed from all the measurements of other tracks and marks on the film.

In the data analysis phase of experiments, the computer is used essentially for three tasks (Rosenfeld and Humphrey 1963). The first is geometrical reconstruction. From the experimental measurements, calculations are made which produce quantities defining the geometry of each event recorded (directions, curvatures and three-dimensional coordinates defining all the measured particle trajectories).

The second is kinematic calculations. Combining the geometrical data with physical data such as particle masses, magnetic field strength, etc., calculations are made to determine kinematic quantities defining the event, such as the momenta and energies of the particle. Least squares fitting procedures are often used to test the fit of the data to alternative physical interpretations of an event within the restrictions implied by conservation of energy and momentum. These calculations usually result in a single physical interpretation being attributed to each event with a certain probability or error.

The third is statistical analysis of the data. The sum total of the geometric and kinematic information for all the events in an experiment (usually thousands) is collected on one or several magnetic tapes and examined by a variety of computer programs. Correlations between computed quantities may be searched for, to indicate some characteristic of the class of event being analysed, or to see whether the data contains biases or systematic errors. Quantities computed for individual events are combined and further calculations made to estimate the final physical results of the exporiment. In this way the computer provides a very powerful means of examining, from many view points, the ensemble of information obtained during an experiment. It is at this stage that the final 'end product' is produced, i.e. physical results such as interaction cross sections, particle angular distributions and life-times, etc., together with estimations of the errors on the quantities measured. Further calculations may be made by using the measured parameters, perhaps combined with results from other experiments, to test the validity of a theoretical model of the process under investigation, or to derive further quantities such as, for instance, particle quantum numbers.

An interesting feature of high energy physics research is the way in which many widespread small research laboratories, particularly in universities, have been able to undertake active research in this field using data obtained from the large accelerator laboratories. This has most readily been achieved for bubble chamber experiments where, once the film has been taken at the accelerator, its measurement and the necessary computing can be done elsewhere. A similar development is beginning to occur for spark chamber and counter experiments with the use of small on-line data acquisition computers to record data on magnetic tape during the experiment at the accelerator. These tapes may then be distributed to many laboratories for analysis.

A very important element contributing to this mode of operation has been the development of a few standard analysis programs which have been used very widely. Considerable effort is required to prepare and continually improve the complex programs needed in the analysis of bubble chamber experiments. A complete chain of programs from the input of measurement data through geometry and kinematics to final analysis may represent more than a hundred thousand 
words of computer code. $\uparrow$ It has been possible, by paying careful attention to programming techniques, to simplify the adaptation of standard programs for operation on a large variety of different computers and for the analysis of all kinds of nuclear events. In addition by providing adequate updating facilities, it has been possible to establish a few program libraries which are now used very widely.

The analysis phase of an experiment is the stage when most computing is done, and it is this which makes high energy physics a very large user of scientific computing capacity. The complete analysis of a nuclear event may require anything up to three or four minutes of computing on a computer such as an IBM 7090.

Generally speaking, the ways in which computers are applied to the planning and data analysis phase of experiments are not particularly specific to his energy physics, but are quite general numerical computing techniques. Similarly, the use of computers in the accelerator or bubble chamber control functions mentioned is very similar to their use in industrial process control applications. We shall therefore not consider these uses in any more detail and the rest of this summary will be concerned with the use of computers for data acquisition and film measurement in high energy physics. As analogue computers are very seldom used in this field, due to their comparatively low precision and limited flexibility, we shall be discussing the use of digital computers only.

\section{Film measurements}

The procedure for examining film is usually divided into two stages, pre-scanning and measuring. During the first the film is examined picture-by-picture on prescanning tables-projectors giving a life-size image on an opaque white screen - to note the serial numbers of those photographs showing events of interest. Depending on the type of experiment being done, interesting events may be found every few to every few hundred photographs. The film is then taken to a measuring device where measurements are made of the events in the photographs selected during the pre-scanning. The reason for this split is essentially one of economics; it is better to do the time-consuming looking at films on relatively cheap prescanning tables, keeping the expensive measuring machines busy as much as possible just measuring.

As can be seen from figures 12 , the quantity and precision of the information in bubble chamber and spark chamber photographs are clearly different. The bubble images on the film are $20-60 \mu \mathrm{m}$ in diameter and their centres can be estimated to about a tenth of a diameter; there are many bubbles along each track so it is possible to define points on the images of the particle tracks to an accuracy of a few micrometres. Also, even on a bubble chamber photograph that contains an interesting event, there are many tracks of no interest, some of which may even obscure those which are of interest. In contrast, the images of sparks are relatively large (about $300 \mu \mathrm{m} \times 30 \mu \mathrm{m}$ wide) and there are only perhaps 20-100 sparks along a track; therefore, each spark defines the track to 0.2 or $0.3 \mathrm{~mm}$. As spark chambers can be positioned at several points along particle trajectories many metres in length, they can yield very precise measurements of these trajectories. In addition, as the spark chambers can be triggered, and as they have very short sensitive times, the photographs show relatively few tracks most of which are

$\dagger$ For instance the CERN bubble chamber analysis programs occupy from 130 to 170 thousand words on a CDC 6600 computer, made up as follows: THRESH (geometry) 38000 , GRIND (kinematics) 33000 , SLICE and SUMX (statistical analysis) from 60000 to 100000 words. part of the event. These characteristics have led to some differences in the ways of measuring spark and bubble chamber film. Due to the lower precision that is often sufficient for spark chamber measurements, the measuring machines can be made simpler and cheaper. The measuring and prescanning can then often becombined into one operation.

Attempts to overcome the slowness of manually operated measuring machines have led to the development of several automated measuring systems involving the use of computers. It has been possible to reach a much higher degree of automation in the measurement of spark chamber films than for bubble chamber films because of the relative simplicity of the photographs and, in some cases, the lower precision required for measurements of spark chamber films. In most spark chamber experiments this higher degree of automation is essential because of the very large number of events recorded (typically over 100000 ).

\subsection{Manual measuring devices}

Basically a manual measuring device requires an operator to make a coincidence between a reference mark and the point to be measured on the photograph. Then by pressing a footpedal the operator can cause the coordinates of the point to be punched on paper tape or cards. This sequence of operations is repeated until all the relevant points on the images of the tracks of an event, as well as the required fiducial marks, have been measured on all three views. The operator usually also has a keyboard via which appropriate identification data (e.g. serial number of the photograph) can be punched on to the output medium. These measurements on the punched tapes or cards can then be read into a computer for analysis by geometry and kinematic analysis programs.

There are two types of manually operated measuring devices in general use today, the projection microscope and the image plane digitizer. The projection microscope consists of a measuring stage or carriage which supports the film (or projection lens) and which can be moved on precision bearings in two perpendicular directions by lead screws or hydraulic rams. An optical system projects the image of the photograph on a screen which carries a reference mark. A digitizing system (e.g. rotary encoders on the lead screws, or moiré fringe digitizers directly on the carriages) together with suitable electronics allows the digital measurements of the stage position to be recorded (digital read-out). These devices can be very complicated (and correspondingly expensive) providing for measurement to a few micrometres precision over image sizes up to $70 \mathrm{~mm} \times 200 \mathrm{~mm}$, projection of three or four views, automatic film transport mechanism, servocontrolled track image following features, etc. (Bradner 1960). Projection microscopes, however, are very slow, measuring only a few events per hour, and are limited in reliability. A typical bubble chamber event might entail measuring between two and three hundred points, which at about $5 \mathrm{~s}$ per point leads to some 20 min per event. The equipment is complicated and mechanical failure of punches and encoders is not infrequent. The operators make errors in the sequence of measurements and, by making errors in aligning the reference mark with the point to be measured, they can also much reduce the accuracy of the measurements. The output is usually only processed at daily intervals on a computer so it is often possible to have errors occurring for very many hours of operation before they are detected.

$\$$ However, it should be noted that there are now many spark chamber experiments where de-magnifications of 50 to $100 \times$ are used in the photography. In these cases measuring devices with precisions of a few micrometres are needed. 
The image plane digitizer consists of a simple coordinate measuring system with digital read-out fitted to scanning tables to make measurements on the projected image rather than the film itself. This class of measuring device of course has a lower precision $(20-100 \mu \mathrm{m}$ on the film) but for some classes of events this precision is acceptable. For such events a significantly higher measuring rate can be achieved (up to 50 events per hour) in comparison with the projection microscopes. Image plane digitizers are used extensively in the measurement of the film from heavy liquid bubble chambers (i.e. those containing, for example, propane). Their accuracy is satisfactory for this type of picture because the multiple scattering that particles undergo in heavy liquids much reduces the intrinsic accuracy with which particle trajectories can be determined.

At the time of the first spark chamber experiments, image plane digitizers were employed to measure the photographs; they were the fastest measuring device available and their precision was adequate for these experiments. However, even though their speed and accuracy may be regarded as adequate for some types of experiment, image plane digitizers still suffer from the same operational deficiencies of low speed and reliability, as do projection microscopes. Efforts to overcome these have led to the development during the last eight or nine years, of new kinds of manually operated measuring systems, as well as the more automatic systems described in the next section. We speak now of measuring systems rather than devices, as the measuring process more and more depends on a device plus one or more computer programs.

There have been two kinds of development of the manually operated measuring systems. The first is the direct connection of a number of measuring machines of the types already described on-line to a small computer (Taft et al. 1964, Bragger et al. 1964, Derrick 1964). The computer programs can 'guide' the operators through the necessary sequence of measurements, at the same time check the accuracy of the measurements. Errors detected can then be immediately corrected by the operators re-measuring some part of the event. The second development has been the construction of new types of projection microscopes and image plane digitizers which incorporate some precision scanning head and which are again operated on-line to a computer. The operator either has to set the reference mark precisely only to a single point (i.e. the vertex) or has to move the reference mark only roughly along the tracks and fiducial marks to be measured. The optical-mechanical scanning head then automatically scans an area of the film around the reference point setting. Some of these devices such as the spiral reader (Burkhard et al. 1965, Alvarez 1962) and the scanning-measuring projector (Hulsizer et al. 1966) scan fairly large regions of the film so that the measurements they make include many points not necessarily on the tracks of interest. Pattern recognition programs (see §2.3) are then used to select the valid measurements. These devices are now coming into use in a few laboratories and measuring rates typically 30-60 events per hour can be achieved.

\subsection{Automatic measuring devices}

A number of film measuring systems have been developed using a variety of automatic measuring devices to examine the film. These are all based on the idea of scanning the film by forming an image (10-25 $\mu \mathrm{m}$ diameter) of a small spot of light on the film and providing a means of moving this spot over the film in such a way that its position is known at any time. A photomultiplier behind the film measures the amount of light transmitted; if the spot falls on a track image the photomultiplier will detect the difference in the light transmitted through the film compared with the case when the spot falls on an area with no image. Since the coordinates of the spot are known, it is possible to measure the coordinates of the track images on the film. There are four main kinds of scan being used at present.

In the area raster scan, an area of the picture is scanned by a spot moving, in sequence, along a number straight parallel lines. The position, size, orientation of the scan area and the number of scan lines in the area can usually be varied (van der Lans 1967). A special case of this type of scan is that where the whole frame is scanned by parallel lines, much like a TV scan. In this case only two orthogonal directions are provided for the scan-line (Anders et al. 1963). In a line scan the spot is scanned along a single straight line, every time the device is instructed to execute a scan. The position, length and orientation of the line can be variable (Bordner et al. 1966). The point-by-point scan is in fact not a true scan of the picture but rather a point-by-point interrogation of the film by the spot. Successive positions for the spot are chosen depending on what was found at previous positions (Deutsch 1965). An area scan by a line segment is similar to the area raster scan with the difference that the scanning is done by a small line segment (typically $1 \mathrm{~mm} \times 25 \mu \mathrm{m}$ ) rather than a circular spot. As the orientation of the line segment is variable, it is possible with suitable discriminators to distinguish segments of tracks, measuring not only their positions but also their directions (Pless et al. 1964).

These scans are generated in two ways: optical-mechanical devices (Benot et al. 1968) that are usually limited to scanning the entire picture with a raster scan, or precision cathode-ray tubes (van der Lans 1967, Anders et al. 1963, Bordner et al. 1966) which can be used to generate all of the various types of scan. The optical-mechanical devices offer the best precision and stability, but produce a large amount of data at each scan (for instance up to 80000 coordinates from a typical bubble chamber picture of which only about $10 \%$ belong to the tracks to be measured). The cathode-ray tubes provide faster scans and, since they can be instructed to scan only selected parts of the pictures, it is possible to reduce the amount of uninteresting data sent to the computer.

These scanning devices are operated on-line with computers. Most of them are intended to run automatically, in the sense that their operators do not normally intervene directly in the measuring process. However, some measuring systems using cathode-ray-tube scanning devices have been built with facilities that are intended to enable their operators to intervene rapidly whenever the system encounters a difficulty (Hodges 1967). The operator is provided with displays of the picture being measured, the activity of the scanner and with a means of accepting or rejecting particular measurements. The advantages of such a system lie in the economies possible in program size and the development time necessary to bring the programs to a point where an acceptably small fraction of the measured events fail to pass completely through the subsequent data analysis programs.

Film measurement for spark chamber experiments using these kinds of techniques has been in regular operation in some laboratories for about five years; measuring rates up to a thousand photographs per hour can now be regularly achieved. For bubble chamber experiments the application of these ideas has proved much more difficult and it is only in the last two or three years that regular and reliable operation has been achieved. Measuring rates fall typically in the range of 30-100 events per hour. Because there are many spark chamber experiments with only few sparks per event and not requiring high measuring precision, a number of laboratories have been developing an essentially visual recording technique that both eliminates the film and combines measuring with 
recording. In this technique, each time the chambers are triggered, two views of the chambers are rapidly scanned by a vidicon. Each time the vidicon senses a spark, the scan line number and the spark's position along the scan line are digitally recorded and this information is either transmitted directly to an on-line computer or recorded on magnetic tape (Macleod and Maglic 1964).

\subsection{The rôle of the computer}

As the measuring devices described above have been developed and as computers have become relatively cheaper and more powerful, increasing advantage has been taken of the possibilities they offer. They have become much more a part of the measuring system and most of these automatic devices could not have been conceived without a suitable computer being available. The rôle of the computer in these measuring systems can be split, broadly, into four tasks, namely data input, control, data checks and data reduction.

Connecting measuring devices directly on-line to computers eliminates the use of an intermediate storage medium (e.g. punched cards) between the device and its computer. Besides removing a major source of failures from the system, a significant amount of card (or paper tape) handling and book-keeping is avoided. For many machines the on-line connection is essential because of their high peak data rates and large quantities of data (Benot et al. 1968), or because they rely on rapid responses from the programs running in the computer (Hodges 1967). In most of the measuring systems described above the computer has the task of controlling various of the actions of the measuring device itself. Some examples are: controlling the film advance equipment; interpretation of the serial number read directly from the picture; controlling the positioning and movement of measuring stages; selecting scan parameters; automatic measurement of fiducial marks, etc. In some systems much of the control logic of the measuring device itself is now provided by programs in a small computer rather than special purpose electronics.

After the data has been read into the computer, the validity of the data is checked in various ways. This is particularly important in the on-line projection microscope and image plane digitizer systems (Taft et al. 1964, Bragger et al. 1964, Derrick 1964). In these systems, the checks range from fitting a curve through the measured points for each track and examining their scatter about the curve, to as far as checking whether the tracks can be satisfactorily reconstructed in space. The data produced by all of the automatic scanning devices, has to be processed by pattern recognition and/or filtering programs, before it is in a suitable form for input to the geometry program.

The task of the filter program is to extract from the data those measurements of points that really correspond to the tracks of interest, fiducial marks, etc. Various techniques are used, making use of the characteristics of tracks such as their continuity, the fact that they can be approximated to by circles or parabolae or that they are roughly radial from the vertex, etc. Depending on the type of device and computers available these filter programs may be run either on-line with the measuring machine, the filtering being done between the measurements of tracks or frames, or off-line (possibly on a different computer) after the measurement of a batch of events. The filter programs need to be told roughly where the required tracks and fiducial marks are located (or that the data mainly contains measurements on these tracks and fiducials). This guidance can be given by making rough measurements with an image plane digitizer on the tracks during pre-scanning. The other extreme is not to provide any guidance but to use much more complex programs able to carry out the entire process unaided. Various intermediate systems exist (Hodges 1967) that use some guidance in conjunction with pattern recognition. In some cases this guidance to the programs is provided by the operator of the measuring device when the programs get into difficulties or are seen to have made mistakes.

For complete pattern recognition the problem is to find all the tracks contained in the measurements of a frame and then to decide which tracks belong to an event. This is to be done without any guidance, except, perhaps, the information that there is an event in the picture. The problem of pattern recognition for spark chamber photographs is significantly easier than for bubble chamber photographs. The amount of data to be processed in these pictures is at least an order of magnitude less; because of their short sensitive times there are few if any background tracks; the sparks are necessarily within well defined areas of the photograph; most pictures will contain an event. Pattern recognition systems can be divided into two broad categories. In the first the complete picture is measured once by a raster scan device and then these measurements are held in the computer memory during part or all of the processing (Marr and Rabinowitz 1966, Zanella 1966). In the second, the photograph is essentially used as a store. An area of the picture is scanned by an area scan device (Yamamoto 1967) or a point is examined (Rudloe et al. 1963); then depending on what is found, for instance a segment of track that matches in position and direction a part of a track that has already been found, the measuring device is instructed to examine another part of the photograph.

In many systems the computer has in addition the task of providing the operator information in as easily assimulated manner as possible, so that he can rapidly provide assistance to the programs, change program parameters, adjust the measuring device, etc. This is done by means of typewritten messages and cathode-ray-tube displays of the picture and of the results of the processing of the picture.

2.4 Future trends

The large bubble chambers of tens of cubic metres capacity (Lentz 1967) which will be coming into operation in the early 1970 's, together with the development of new types of optical spark chambers (e.g. wide-gap and streamer chambers (Odian 1966, Alikhanian 1966)) will certainly entail further development of the kinds of computer-based film measuring systems outlined above. New types of measuring devices may be introduced (such as for example laser scanners or returnbeam videcons) to obtain higher speeds, or precision of measurement, or more flexibility for operation under control of computer programs. The next few years may well see developments undertaken towards direct conversion of bubble chamber images into digital form in real-time.

We can expect to see a closer integration of measuring device, computer and operator being achieved. The human operator can probably still examine bubble chamber film and find events more economically and with greater flexibility than can a computer with a pattern recognition program. Therefore systems will be developed that are aimed at providing the operators with the maximum support possible; the measuring device and computer will carry out all of the routine tasks, in order to make best use of the operator's intelligence for difficult decision processes. As remote computing and computer display techniques become more developed they will find application in this area; visual comparison of the film and the measurements may be made in real time as the computer analyses the data. Further development of these ideas may lead to measurement centres being set up equipped with large computer facilities and which 
would be used as 'data banks' for many distant physics laboratories.

Another area where important developments may be expected is in pattern recognition. In addition to the continuing refinement of programming techniques there is the development of special purpose computer processors, designed specifically to aid the solution of the problem of pattern recognition in bubble chamber pictures, e.g. the SATR project (Thompson 1967) where special arithmetic units and a so-called ' $3-\mathrm{D}$ register' are being added to a large computer. The arithmetic units will provide the transformation from the coordinates of a point in the film plane to the coordinates and directions of the corresponding light rays in the bubble chamber. The 3-D register is to be used to represent a small volume of the chamber and to discover whether any of the light rays intersect within that volume and then whether these intersections represent a segment of a track. In an even more elaborate project, Illiac III (McCormick and Nansimhan 1963), a special processor has been built, that can carry out, in parallel, topological transformations on patterns represented by bits in a $32 \times 32$ array of registers.

As the number of events measured per year in laboratories rises, a new problem is being encountered, i.e. the bookkeeping and organizing required to control all the various stages in the measuring and analysing chain, the numerous magnetic tapes generated between stages in the processing, the remeasurement of rejected events, etc. Here too computers can be of help, by providing systems for mass data storage with efficient indexing schemes and multi-access systems to enable the physicists to organize more readily the statistical analysis of the events measured for their experiments. Presently available mass store sizes (up to $10^{8}-10^{9}$ bits) do limit the extent to which developments in this direction can be made; techniques are expected to improve so that mass stores of $10^{11}-10^{12}$ bits with access time of a few seconds will be available.

\section{Data acquisition}

\subsection{Outline of the technique}

In those experiments where film is not used as a recording medium - loosely referred to here as counter experiments the process of presenting the recorded data in a form suitable for computer analysis is in many ways simplified. Scintillation or Cerenkov counters and counter hodoscopes are all essentially digital devices. They record a binary signal indicating the presence (or absence) of a particle. The analogue information they may provide, such as their geometrical position which defines a physical volume through which the trajectory of the particle passes, is readily available in digital form - for instance simply by interrogating them in a known order.

The development of spark chamber techniques has been strongly influenced by the wish to take advantage of their good spatial resolution in counter experiments while avoiding the intermediate system of photographic recording and film measurement. Various read-out systems (sonic, ferrite core, magnetostrictive and current division (Wenzel 1966)) have been developed to produce a directly digitized output of the spark coordinates.

The use of digital computers in the analysis of data recorded by counter techniques has two particular advantages. The first is that the computer can be used as a very versatile logical element in deciding whether or not the signals recorded by the counters correspond to the occurrence of the type of nuclear event being examined. The second is their use on-line which provides a feedback of processed information to the experimentalist during the course of the setting-up and data taking runs at the accelerator.

Historically the early efforts to use computers in counter experiments were directed towards the first of these. Recording devices, typically a buffer memory feeding a digital magnetic tape recorder (Lindenbaum 1963) were used to record, on magnetic tape, counter information. Subsequently the data on the tapes was processed by a computer, which was able to select by various calculations the data corresponding to the events being looked for. Then for these events more detailed analysis calculations would be made. This procedure has an advantage over earlier techniques that the selection of events to be analysed from background events may be made after the data is recorded by using a computer to make tests based on calculations exploiting, say, the kinematic properties of the events. This allows the data-taking run at the accelerator to be made with much looser triggering criteria. This not only reduces the risk of biases in the data, but also raises the absolute rate of recording interesting events or, alternatively, permits the recording of different types of event within one run at the accelerator.

The advent of the small fast digital computer at prices comparable to the cost of other electronic equipment used in physics experiments, made possible the rapid development of the on-line use of computers in counter experiments. Standard magnetic tape peripheral equipment can be used for permanent storage of the data ready for subsequent analysis on a larger computer. The small computer can be programmed to carry out many logical tests for internal consistency on the data and to print values of significant parameters for the experimentalist. The computer can also be made to carry out various logkeeping tasks during the accelerator run, and to indicate via a printer any indications of malfunctioning of the equipment. The speeds of calculation available are such that these operations can be carried out as the data is being accumulated, and on pulsed accelerators often between pulses. The whole procedure is very flexible in that program changes can be made in the light of experience much more readily than changes could be made if these functions were carried out by special purpose electronics. For instance, program changes can alter the logical tests applied to the data, or can modify the calculations to be made or the kinds of data logged, etc. This whole procedure available to the experimentalist is a very powerful aid in setting up and monitoring the progress of the data acquisition phase of an experiment. In some cases, where the on-line computing capacity and the nature of the experiment allows, it is also possible to carry out a more complete analysis of some of the data during the accelerator run. These sample calculations may represent the complete analysis to be made on the experimental data, but performed for some fraction, say a few per cent, of the total data recorded. This provides a running check on the nature and quality of the data recorded and provides a further means of ensuring efficient control over the progress of the experiment.

3.2 The on-line computer and data rates

The characteristics of computers used on-line in counter experiments vary somewhat depending upon the needs of the experiment, but by-and-large they can be summarized as follows. The memory may be 4,8 or 16 thousand words of 12-24 bits and with a cycle time of a few microseconds or less. The processor should provide fast fixed-point arithmetic and logical operations. A flexible input-output arrangement is necessary with one or more direct memory access channels and a multi-level interrupt facility. Peripheral equipment may consist of one or two magnetic tape units, a teleprinter or slow line printer, punched card or paper tape reader and punch, and possibly a graphical display unit. Small disk memories, typically half a million words, are also coming into use because of the greatly enhanced programming flexibility they provide. The programming is mostly done in assembly language both 
because the real-time operation requires very careful timing of input and output, and also because the limited memory requires that programs be as small as possible. A higher level language such as Fortran is often used in the early stages of an experiment when the feasibility of analysis methods is being tested and changes are frequently required.

The rates at which information may be recorded in counter experiments depends upon many factors. The accelerator beam intensity, pulse length and repetition rate, the nature of the events under study, the triggering rate of the detection system, the number of counters and spark chambers involved and their dead-times all play a rôle in determining the data rates achieved.

The synchrotrons and linear accelerators used for high energy physics have pulsed beam characteristics; typically a synchrotron pulse may last $200 \mathrm{~ms}$ and be repeated every few seconds. In an experiment using wire spark chambers and counters events may be detected at rates of up to 200 per second during the $200 \mathrm{~ms}$ accelerator pulse (i.e. up to 40 events per pulse) the limit being set by the spark chamber dead-times. This would be a typical maximum or peak rate; in practice average rates may be somewhat lower, depending upon the beam intensity and kind of event being studied. If the detectors are all counters then much higher rates can be achieved, but practical limits are soon set by the speed or size of the memory of the on-line computer, though for simple events rates of 1 million per hour have been achieved.

The amount of information recorded for an event varies very much from experiment to experiment. Counter arrays up to several hundred elements have been used (Foley et al. 1964). Each counter signal then needs nine or ten bits to specify it. The largest spark chambers used so far have been about $1 \mathrm{~m}$ square with a spatial resolution of $1 \mathrm{~mm}$ in each of two coordinates, requiring ten bits for each coordinate. An experiment may use ten or twenty spark chambers, and as many counters. Assuming an average of 10 events are recorded per pulse, and that each event is defined by 20 pairs of ten-bit coordinates from the spark chambers plus five-bit numbers from each of 20 counters, the data per pulse to be stored reaches 5000 bits, or 600 computer words if one coordinate is stored per word. This kind of example is quite typical and although practical data rates used vary very considerably depending on the kind of experiment, they generally fall in the range of a few hundred to a few thousand computer words during an accelerator pulse, the whole repeated every few seconds.

\subsection{Program organization}

The computer programs used are organized around the interrupt facilities of the computer. These correspond to electronic signals sent from the counter and spark chamber equipment to the computer to indicate certain conditions and each such interrupt causes the computer program to stop its current activity and pass to a special sub-program which identifies the kind of interrupt condition. Then, according to a preset table which lists the priority to be accorded to each kind of interrupt, the program decides what action to take.

The primary function of the computer used on-line is to record data. The highest level priority will therefore be given to an interrupt caused by the signal corresponding to the detection of an event. The response to this interrupt would normally be within, at most, a few tens of microseconds, by which time the computer would begin to read in the information from the counters and spark chambers. A part of the computer memory is usually set aside as a buffer area to receive the data recorded during the accelerator pulse. The transfer would be completed in a few milliseconds or less. The computer can then proceed with the next lower level priority task. After the elapse of their recovery times and the re-setting of the various counters, the equipment would be ready to record the next event, which, when it occurred, would again generate the highest priority interrupt and the process would be repeated. Between accelerator pulses the computer program transfers the data accumulated in a buffer on to magnetic tape; this is the permanent storage of the 'raw' data. The computer will use up whatever processing time it has left in various lower priority activities. Internal consistency checks might be made on the data in the input buffer (for instance, if three separate counter arrays signal the passage of a particle a test could be made to see that the position given by the central one is consistent with a prediction from the positions given by the first and last). Various statistics would be kept, such as the number of counts from individual counters or the number of events recorded per pulse. From time to time a summary of these would be printed out for logging purposes. If some statistics are obviously incorrect, or the internal consistency checks fail, the computer would print out a warning message.

The experimentalist has the possibility of interrogating the computer, usually via a keyboard or switches, to ask for printed summaries of information stored in the machine, or to cause particular programs to be executed to make a specific analysis on some of the recorded data. He can also type in information to the computer such as date and time, changes of experimental conditions, etc. All these facilities are ained at using the computer to provide information on the progress of the experiment and the proper functioning of the apparatus. The communication with the experimentalist is facilitated if a display device, such as a cathode-ray tube, is used. Firstly, some information can be rapidly displayed for a quick survey, selecting only what is really needed for subsequent (slower) output on a typewriter. Secondly, much information can be displayed in a graphical form rather than in tables for instance a histogram of the statistical information kept is often much more readily assimilated and understood by the experimentalist than a table of numbers. Thirdly, if a light pen is provided, a very flexible and powerful means of input to the computer is provided - for instance a region of a histogram can be selected, just by pointing, for enlargement. Considerable work is going on at present to develop these kinds of techniques in engineering and computer sciences as well as in high energy physics (van Dam 1966).

3.4 Future trends

As an extension of its rôle in checking equipment operation, the on-line computer is beginning to be used to assist the experimentalist by carrying out a number of more complex but routine measurements. By providing appropriate analogueto-digital converters, control electronics and programs, the computer can be made to check constantly such items as magnet currents, photomultiplier voltages, etc.; the computer can adjust such parameters to the correct values. Such routine but time-consuming procedures as measuring beam intensity or plotting beam profiles and counter response curves can be carried out by the computer, and several laboratories are engaged in developing on-line computer techniques in this direction.

A further important trend is to increase the computing capacity available by the connection of one or several small on-line computers to a larger multiprogramming computer (Fannin 1967). It is usually too expensive, and therefore rare, that the on-line computer itself has enough computing capacity to do more than record the data and make the kind of checking operations described above. However, it can be of great assistance whilst setting up the best operating conditions for an experiment to be able completely to analyse a 
small amount of recorded data, and to observe the effects of changing certain experimental conditions or certain program parameters, on the fully computed physics results. This can be achieved by arranging with the laboratory's main computer centre to have, every hour or so, a short run on a large computer to process some raw data recorded on magnetic tape. Such an arrangement is quite well suited to routine checking of the data being accumulated during a long accelerator run, but not very suitable to the requirements during the setting-up period when a rapid response is needed as conditions change. Connection of the small computer to a large one via low or medium speed data link allows direct transmission of raw data to the large machine for detailed analysis. Rather complex programming arrangements have to be made to allow the computers to communicate effectively one to another, to ensure processing of the data run after it is transmitted, and return of results. The flexibility of these arrangements is much enhanced if the experimentalist is able to communicate with programs in the large computer in order to be able to use or modify one of a number of different programs to make different analyses of the data. The practical limits for this kind of operation, which in many ways can be regarded as a special case of a general development towards time-shared use of computers, are set by either the cost, or the presence of other work on the large computer which limits the capacity available for the on-line user.

References

Alikhanian A I 1966 Proc. Int. Conf. Instrumentation for High Energy Physics, Stanford, California 1966 (Stanford: Stanford Linear Accelerator Center) p. 415

Alvarez L W 1962 Proc. Informal Meeting on Track Data Processing, CERN, 1962 ed. M Benot and B Elliott, CERN publication no. $62-37$ p. 43

Anders H et al. 1963 Proc. Int. Symp. Nuclear Electronics Paris, 1963 (Paris: La Société Française des Electroniciens et des Radioélectriciens) p. 349

Benot M et al. 1968 The HPD Mark 2 Flying Spot Digitizer at CERN, CERN publication no. 68-4

Bordner C A et al. 1966 Proc. Int. Conf. Instrumentation for High Energy Physics Stanford, California, 1966 (Stanford: Stanford Linear Accelerator Center) p. 661

Bradner H 1960 Ann. Rev. Nucl. Sci. 10149

Bragger H R et al. 1964 Proc. Int. Conf. on High Energy Physics, Dubna 1964, Vol. 2 (Moscow: Atomizdat) p. 393-4

Burkhard J H et al. 1965 Proc. Conf. Programming for Flying Spot Devices, Columbia University, 1965 Vol. I (New York: Columbia University) p. 2.1

van Dam A 1966 Adv. Computers 7239

Derrick K 1964 Proc. 12th Int. Conf. High Energy Physics, Dubna, 1964 ed. Ya A Smorodisky Vol. 2 (Moscow: Atomizdat) p. 395-7

Deutsch M 1965 IEEE Trans. Nucl. Sci. NS-12 69

Fannin K R 1967 Proc. AEC Computer Informal Meeting, Rice University, Houston 1967 AMD 456, BNL 11277

Foley K J et al. 1964 Proc. Informal Meeting on film-less Spark Chamber Techniques and Associated Computer Use ed. G R Macleod and B Maglic CERN publication no. 64-30 p. 11

Hodges D 1967 Proc. Conf. on Programming for Flying Spot Devices, Munich (Munich: Max-Planck-Institut) p. 325
Howard F T 1967a IEEE Trans. Nucl. Sci. NS-14 No. 3

Howard F T 1967b Proc. 6th Int. Conf. High Energy Accelerators, Cambridge, 1967 (Cambridge, Mass: Cambridge Electron Accelerator) Appendix B

Howry S K 1967 IEEE Trans. Nucl. Sci. NS-14 1066

Hulsizer R J et al. 1966 Methods in Computational Physics Vol. 5 (New York: Academic Press) p. 158

James F 1968 Monte Carlo Phase Space CERN publication no. $68-15$

van der Lans J 1967 Proc. Conf. Programming for Flying Spot Devices, Munich, 1967 (Munich: Max-Planck-Institut)

Lentz H 1967 Proc. Int. Colloquium Bubble Chamber, Heidelberg, 1967 CERN publication no. 67-26, Vols I and II

Lindenbaum S J 1963 Nucl. Instrum. Meth. 20297

Macleod G R and Maglic B (eds) 1964 Proc. Informal Meeting on Film-less Spark Chamber Techniques and Associated Computer Use CERN publication no. 64-30 p. 57

Marr R B and Rabinowitz G 1966 Meth. Computational Phys. 5213

McCormick R H and Nansimhan R 1963 Nucl. Instrum. Meth. 20401

Odian A 1966 Proc. Int. Conf. Instrumentation for High Energy Physics, Stanford, California (Stanford: Stanford Linear Accelerator Center) p. 49

Piroué P 1966 IEEE Trans. Nucl. Sci. NS-13 1

Pless I et al. 1964 Proc. 12th Int. Conf. High Energy Physics, Dubna, 1964 Vol. 2 (Moscow: Atomizdat) p. 409

Ritson D M (ed.) 1966 Proc. Int. Conf. Instrumentation for High Energy Physics, Stanford, California, 1966 (Stanford: Stanford Linear Accelerator Center) Sessions A, B, I, K and $\mathrm{L}$

Rosenfeld A H and Humphrey W E 1963 Ann. Rev. Nucl. Sci. 13 106-29

Rudloe $\mathrm{H}$ et al. 1963 Communications of the A.C.M. 6332

Simpson J D 1967 Proc. Int. Colloquium on Bubble Chambers, Heidelberg, 1967 ed. H Leutz CERN publication no. 67-26 Vol. II p. 501

Taft H D et al. 1964 Proc. 12th Int. Conf. on High Energy Physics, Dubna, 1964, Vol. 2 (Moscow: Atomizdat) p. $390-2$

Thompson M A 1967 Proc. Conf. Programming for Flying Spot Devices, Munich, 1967 (Munich: Max-Planck-Institut) p. 366

Wenzel W A 1966 IEEE Trans. Nucl. Sci. NS-13 34

Whiteside D and Gardner J W 1963 Nat. Inst. Res. Nucl. Sci. NIRNL/M/44

Yamamoto R K 1967 Proc. Conf. Programming for Flying Spot Devices, Munich, 1967 (Munich: Max-Planck-Institut) p. 314

Zanella P 1966 Proc. Int. Conf. on Instrumentation for High Energy Physics, Stanford, California, 1966 (Stanford: Stanford Linear Accelerator Center) p. 519 